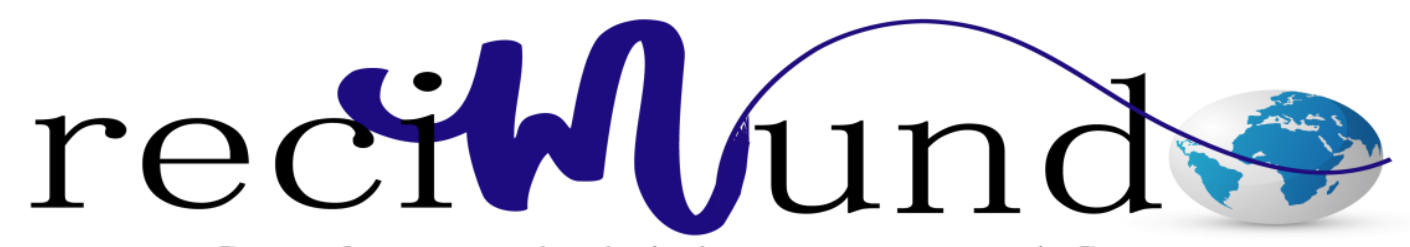

Revista Científica Mundo de la Investigación y el Conocimiento

Felix Cristobal Hablich Sánchez ${ }^{\text {a }}$ Ingrid Irene Toala Rocuano ${ }^{\text {, }}$ Mercy Victoria Agila Maldonado ${ }^{c}$

Las empresas públicas con economía mixta en el mercado de valores en el Ecuador

Revista Científica Mundo de la Investigación y el Conocimiento. Vol. 2 núm., 1, febrero, ISSN: 2588-073X, 2018, pp. 784-799

DOI: $\underline{10.26820 / \text { recimundo/2.1.2018.784-799 }}$

Editorial Saberes del Conocimiento

Recibido: 05/12/2017

Aceptado: 10/02/2018

I. Universidad de Guayaquil.

II. Universidad de Guayaquil.

III. Universidad de Guayaquil. 


\section{Las empresas públicas con economía mixta en el mercado de valores en el}

Ecuador

Vol. 2, núm. 1., (2018)

Felix Cristobal Hablich Sánchez; Ingrid Irene Toala Rocuano, Mercy Victoria Agila Maldonado

\section{Resumen}

La Constitución de la República del Ecuador dispone que el Estado constituya empresa pública para la gestión de sectores estratégicos, la prestación de servicios públicos, el aprovechamiento sustentable de los recursos naturales o de bienes públicos y el desarrollo de otras actividades económicas Tomando en cuenta los procesos de transformación que se han desarrollado en los últimos tiempos, resulta de interés realizar una investigación respecto a las posibilidades reales de que las empresas públicas no solo decidan llevar economías mixtas en donde se pretende una descentralización de las decisiones sino que la economía de mercado, vista desde las posibilidades de inversión en el mercado de valores, muestren alternativas reales de la autogestión, refinanciamiento e independencia económica sustentable para disminuir el gasto público e incentivar la inversión social con miras a la cogestión. A través del análisis crítico de una realidad socioeconómica, el científico social obtiene herramientas de observación que permiten proponer hipótesis económicas. Aunque el Estado ha tenido signos de impulsar el Mercado de Valores, la conclusión respecto a la participación de las empresas públicas en el mismo se centra en la confianza. De haber una fortaleza real del mundo bursátil ecuatoriano, las empresas publicas mismas deberían ser las primeras obligadas a destinar parte de sus rentas al impulso y fortalecimiento del sistema bursátil, pero atendiendo la investigación, la decisión para el razonamiento economicista sería que aún no existe una variable de confianza que apruebe la inversión del capital mixto de una empresa pública en un mercado de valores con las debilidades que muestra.

Palabras Claves: Mercado, bursátil, empresa, publica, capital. 


\section{Las empresas públicas con economía mixta en el mercado de valores en el Ecuador}

Vol. 2, núm. 1., (2018)

Felix Cristobal Hablich Sánchez; Ingrid Irene Toala Rocuano, Mercy Victoria Agila Maldonado

\section{Summary}

The Constitution of the Republic of Ecuador provides that the State constitutes a public company for the management of strategic sectors, the provision of public services, the sustainable use of natural resources or public goods and the development of other economic activities Taking into account the processes of transformation that have been developed in recent times, it is interesting to conduct research on the real possibilities that public companies not only decide to lead mixed economies where the aim is to decentralize decisions but the market economy, seen from the possibilities of investment in the stock market, show real alternatives of self-management, refinancing and sustainable economic independence to reduce public spending and encourage social investment with a view to co-management. Through the critical analysis of a socioeconomic reality, the social scientist obtains observation tools that allow proposing economic hypotheses. Although the State has had signs of boosting the Stock Market, the conclusion regarding the participation of public companies in the same focuses on trust. If there is a real strength of the Ecuadorian stock market, the public companies themselves should be the first ones to allocate part of their income to the impulse and strengthening of the stock market system, but taking into account the research, the decision for economistic reasoning would be that there is still no variable of confidence that approves the investment of the mixed capital of a public company in a stock market with the weaknesses that it shows.

Key Words: Market, stock market, company, public, capital. 


\section{Las empresas públicas con economía mixta en el mercado de valores en el}

Ecuador

Vol. 2, núm. 1., (2018)

Felix Cristobal Hablich Sánchez; Ingrid Irene Toala Rocuano, Mercy Victoria Agila Maldonado

\section{Introducción.}

Quizás el tema más recurrente planteado en la actual discusión sobre la crisis es el de los roles del Estado. Se trata por cierto de un tópico universal, que abarca tanto a las sociedades del centro como a las de la semiperiferia; a las economías centralmente planificadas como a las mixtas. La controversia emerge en los años 70 y se corresponde con la decadencia del llamado Estado de Bienestar en los países capitalistas avanzados y del Estado de compromiso nacional en las sociedades más atrasadas. Los años 80 colocaron a buena parte de los países latinoamericanos ante un desafío particular: la combinación entre la voluntad de construcción de regímenes democráticos y una gran crisis económica, que obliga a redefinir las relaciones entre Estado y sociedad; entre Gobierno y mercado; entre política y economía. Se trata, pues, de una doble oportunidad: la de superar las formas del autoritarismo y la de modificar un tipo de acumulación, que desde finales de los 60 ha perdido su capacidad expansiva. Y ambas tareas pasan centralmente por reformas en el Estado. Así, todo suele resumirse en el enfrentamiento abstracto entre «privatistas» $\mathrm{y}$ «estatistas» sobre un tema también parcial, como es el de la situación de las empresas en manos de los gobiernos. Hacen falta fórmulas innovadoras, originales, imaginativas, capaces de renovar esquemas propios ya perimidos y de enfrentar la ofensiva de la Nueva Derecha. La vía para ello tiene como presupuesto teórico una distinta proyección de las relaciones entre Estado y sociedad. Surge "lo público", entendido como un espacio que pueda asegurar una mayor información, participación y descentralización de las decisiones. Es este crecimiento del poder de la sociedad civil; es este fortalecimiento del espacio público con relación al orden estatal y al orden privado, lo que le permite a la teoría democrática vincular la reforma económico-social del Estado con la reforma política del Estado. Esta opción de ningún 


\section{Las empresas públicas con economía mixta en el mercado de valores en el Ecuador}

Vol. 2, núm. 1., (2018)

Felix Cristobal Hablich Sánchez; Ingrid Irene Toala Rocuano, Mercy Victoria Agila Maldonado modo elimina los roles del mercado, ni subestima los roles decisorios del Estado. Lo que potencia es la reconstrucción de la sociedad ahogada por el centralismo burocrático, tanto como por la mercantilización de todas las relaciones humanas. (Portantiero, 2013, pág. 158, 164 y 167).

El Artículo 315 de la Constitución de la República del Ecuador dispone que el Estado constituya empresa pública para la gestión de sectores estratégicos, la prestación de servicios públicos, el aprovechamiento sustentable de los recursos naturales o de bienes públicos y el desarrollo de otras actividades económicas y el artículo 5 de la Ley Orgánica de Empresas Públicas, los Gobiernos Autónomos, están facultados para constituir, organizar, fusionar y liquidar empresas públicas, mediante ordenanzas que permitan la prestación eficiente de los servicios públicos que son de su competencia. (Bustamante \& Bustamante, 2017, pág. 1).

Los mercados financieros se pueden clasificar en dos principales: monetario y de capitales. El primero, a través del mercado de dinero, tiene como papel principal trasladar el ahorro a la inversión, vía la intermediación financiera, con lo cual capta recursos del público y otorga financiamiento de corto plazo con la participación de bancos, sociedades financieras, cooperativas y otros intermediarios financieros. El mercado de capital tiene como función captar ahorro y financiar inversión, la que se realiza a través del mercado de valores y mercado de crédito de mediano y largo plazo. El mercado de capital juega un papel importante tanto en la asignación de recursos como en el crecimiento económico. (Rosero, 2010, pág. 24).

Tomando en cuenta los procesos de transformación que se han desarrollado en los últimos tiempos, no solo en Ecuador, sino en Latinoamérica, además de la discusión acerca del 


\section{Las empresas públicas con economía mixta en el mercado de valores en el}

Ecuador

Vol. 2, núm. 1., (2018)

Felix Cristobal Hablich Sánchez; Ingrid Irene Toala Rocuano, Mercy Victoria Agila Maldonado alcance o limitación del mercado de capital versus el rol del Estado resulta de interés realizar una investigación respecto a las posibilidades reales de que las empresas públicas no solo decidan llevar economías mixtas en donde se pretende una desentralización de las decisiones sino que la economía de mercado, vista desde las posibilidades de inversión en el mercado de valores, muestren alternativas reales de la autogestión, refinanciamiento e independencia económica sustentable para disminuir el gasto publico e incentivar la inversión social con miras a la cogestión.

\section{Materiales y Métodos.}

A través del análisis crítico de una realidad socioeconómica, el científico social obtiene herramientas de observación que permiten proponer hipótesis económicas. La importancia de este hecho científico recae que, en las ciencias sociales, la investigación positivista no suele dar verdaderas respuestas a la incognita académica sobre la cosa social.

Se ha hecho uso de las investigaciones académicas y las herramientas tecnológicas de información y comunicación para acceder al debate económico respecto al rol del Estado en la Economía, las funciones de las empresas públicas, el manejo mixto del capital corporativo y la participación en el Mercado de valores, delimitado en Ecuador como espacio físico de estudio para identificar una idea global planteado en el título de ésta trabajo.

Haciendo uso de la lectura, resumen y análisis se hace una recopilación bibliográfica no experimental para proponer las conclusiones que hubiera a lugar según la información disponible en revistas científicas, libros y reportes disponibles on-line bajo el reconocimiento académico de la información expuesta. 


\section{Las empresas públicas con economía mixta en el mercado de valores en el Ecuador}

Vol. 2, núm. 1., (2018)

Felix Cristobal Hablich Sánchez; Ingrid Irene Toala Rocuano, Mercy Victoria Agila Maldonado

\section{Resultados.}

A través de los modelos organizacionales se implementa un desarrollo integral para el buen vivir, sustentado a niveles de alta gerencia, administrativo y con el desarrollo de las comunidades, fortalecimiento y creación de nuevas capacidades, facilitando la ejecución de un modelo de desarrollo socio económico a través del establecimiento de una planificación eficiente y la incorporación de una gestión por resultados que logre un desarrollo integral sustentable, permitiendo la optimización de recursos económicos y laborales con que cuentan estas empresas adscritas al Gobierno, propiciando resultados positivos dirigidos a la comunidad, mediante planificación y optimización de recursos del personal de las empresas públicas logrando un modelo de desarrollo organizacional eficiente, inversión en servicio social y en obras prioritarias que permitan mejorar la calidad de vida y el desarrollo humano en los sectores más vulnerables y de atención prioritaria. (Bustamante \& Bustamante, 2017, pág. 1).

Lo que debería seriamente debatirse, en cambio, es la transformación estructural del Estado, en lo económico y en lo político. Esa transformación se refiere, por lo menos, a cuatro áreas de actividad: la administrativa, para mejorar sus rendimientos; la económica, no solo en lo que se refiere a la posibilidad de privatizar total o parcialmente empresas estatales, sino también a la de «privatizar» al capitalismo subsidiado que vive de la protección del Estado; la social, tratando de reestructurar el gasto, minimizando los costos burocráticos y maximizándolos en términos de equidad, para orientarlos hacia las categorías más débiles, reduciendo la ayuda a otras; y por fin, la institucional, que en muchos casos, como el de Argentina, supone la reforma 


\section{Las empresas públicas con economía mixta en el mercado de valores en el}

Ecuador

Vol. 2, núm. 1., (2018)

Felix Cristobal Hablich Sánchez; Ingrid Irene Toala Rocuano, Mercy Victoria Agila Maldonado de una Constitución decimonónica para adaptarla a los tiempos actuales. (Portantiero, 2013, pág. 164 y 165).

La revolución tecnológica en marcha está sin duda hegemonizada por el centro capitalista y hasta las grandes economías planificadas y centralizadas que se diseñaron como alternativa deben subordinarse, para poder salir de su propia crisis. El discurso ideológico que lo nutrió tradicionalmente, que concedía al Estado un rol central como agente de cambio, no se compadece ya con la realidad, entre otras cosas, porque ese Estado está hoy feudalizado por las corporaciones. (Portantiero, 2013, pág. 165).

El mercado de valores es una alternativa muy relevante al mercado del dinero de tal manera que un ahorrista tiene la potestad de invertir el mismo, a través de la compra de una acción, con emisiones de una empresa que le permita tener recursos monetarios para mejorar sus condiciones financieras. En el Ecuador las transacciones totales de la Bolsa de Valores en relación al PIB representan entre el 8.9\% y 9.4\% en el periodo entre el 2006 y 2010. Para el año del 2011 las inversiones se en 5.7\% del PIB por lo que resulta necesario optimizar los incentivos tributarios. (Pérez, Rivera, \& Solís, 2015, pág. 9).

El mercado de valores propenden a la capitalización de recursos financieros, ya que asegura una fuente de financiamiento para las empresas, esto requiere a su vez del concurso del potencial humano y de la tecnología. El mercado de valores ecuatoriano está poco desarrollado, debido a una marcada tradición al financiamiento con deuda por parte de las mismas empresas, es decir los pequeños y medianos empresarios son renuentes a acudir al mercado de valores para 


\section{Las empresas públicas con economía mixta en el mercado de valores en el Ecuador}

Vol. 2, núm. 1., (2018)

Felix Cristobal Hablich Sánchez; Ingrid Irene Toala Rocuano, Mercy Victoria Agila Maldonado financiarse; lo que es un grave problema de democratización de los recursos. (Pérez, Rivera, \& Solís, 2015, pág. 9 y 10).

El gobierno de Rafael Correa ha impulsado la nueva arquitectura financiera regional, aprobada por la Asamblea en Mayo de 2010, mediante la cual se crea el Banco del Sur y el Sistema Único de Compensación Regional (SUCRE) lo cual se presenta como una alternativa al sistema financiero internacional establecido en Bretton Woods, del cual surgieron los organismos financieros internacionales como el FMI y el Banco Mundial. El gobierno actual está planteando una nueva arquitectura financiera interna, cuyo contenido todavía no está claro, pero hay indicios de su construcción con la modernización de la red de seguridad financiera1, las reformas al Banco Central2, la creación del Banco del Instituto Ecuatoriano del Seguro Social, el estudio de la redefinición del rol de la banca pública, el papel del Banco del Pacífico, la nueva ley del mercado de valores, etc. Se estaría apuntando a una reforma financiera, cuyos primeros pasos ya se han dado. (Rosero, 2010, pág. 23).

La expedición de la Ley de Mercado de Valores es uno de los pasos que ha dado el Ecuador, para tratar de insertarse dentro del nuevo proceso de internacionalización de la economía y conseguir la atracción de la inversión extranjera; los mismos que son factores exógenos que ayudaron a dar este significativo paso. En definitiva, los aportes y beneficios que ha pretendido dar la Ley, son una mejor utilización de los instrumentos financieros en el Mercado de Valores, para tratar de permitir una excelente canalización del ahorro, lo que debería fortalecer el sector productivo. En cuanto al crédito otorgado por el Mercado de Valores resulta más eficiente y eficaz que cualquier otra fuente de financiamiento, se adaptan a las necesidades 


\section{Las empresas públicas con economía mixta en el mercado de valores en el}

Ecuador

Vol. 2, núm. 1., (2018)

Felix Cristobal Hablich Sánchez; Ingrid Irene Toala Rocuano, Mercy Victoria Agila Maldonado de financiamiento e inversión y sus posibilidades de pago a tasas de interés que son mayores respecto de la tasa pasiva para los inversionistas y menores que la tasa activa para los emisores, a plazos más prolongados que las que ofrece el sistema financiero tradicional. La propuesta de Ley de Mercado de Valores que está bajo el cargo del Ministerio de Coordinación de la Política Económica, trae consigo nuevas perspectivas, como la potencial democratización de esta actividad y el surgimiento del mercado alternativo (Pérez, Rivera, \& Solís, 2015, pág. 10).

El desarrollo socioeconómico de las comunidades mediante la ejecución de proyectos de desarrollo socioeconómico constituye un valioso aporte hacia la prosperidad y la igualdad social en los diferentes entornos y en los diferentes estratos sociales; por lo general estos proyectos se direccionan a la solución de problemas que de forma directa obstaculizan el crecimiento económico y social de las poblaciones; como es el caso de la falta de sistemas de riego, falta de capacitación en determinado tema de necesidad, falta de una organización comunitaria, falta de servicios básicos, entre otros. (Bustamante \& Bustamante, 2017, pág. 12).

Adicionalmente, se debe indicar que nuestro mercado es sobre todo un gran nicho para la venta de renta fija donde las empresas que han conocido de las bondades del mercado han sabido beneficiarse de tasas de interés más bajas sin costos de garantías y además sin perder el control societario, lo que se debe destacar considerando el altísimo número de compañías familiares en nuestro país. Concomitantemente, los inversores han tenido la oportunidad de confiar de manera segura sus inversiones y obtener más rendimiento por sus inversiones.

Las negociaciones de renta variable son muy escasas y de los emisores que han inscrito sus acciones son muy pocas las que se transan constantemente en nuestras bolsas de valores, tal 


\section{Las empresas públicas con economía mixta en el mercado de valores en el Ecuador}

Vol. 2, núm. 1., (2018)

Felix Cristobal Hablich Sánchez; Ingrid Irene Toala Rocuano, Mercy Victoria Agila Maldonado

como podemos ver por el bajísimo \% de las negociaciones de los últimos años en la Bolsa de Valores ecuatoriana, menos del 5\%. Del total negociado.

En los últimos años ha destacado las negociaciones de valores producto de procesos de titularización y entre ellas de las denominadas titularizaciones de flujos, a las que nos referiremos más adelante. Estos son valores ante todo de renta fija que han sido adquiridos por inversionistas institucionales del sector público y privado, La mayoría de los valores emitidos se presentan todavía de manera física. La desmaterialización de los valores se ha incrementado en los últimos años pero todavía tenemos un mercado en el que prevalece la circulación de documentos impresos, lo que atenta contra la agilidad y seguridad que se obtiene con la desmaterialización. Adicionalmente, es importante recalcar la participación de las instituciones financieras en nuestro mercado de valores, siendo actores permanentes del mismo como emisores ante todo de obligaciones y papel comercial. Sin embargo, estos valores en muchas ocasiones fueron adquiridos por miembros del mismo grupo financiero y que se limitaron a un mercado primario para solamente cumplir requisitos de encaje obligatorio y para cumplir exigencias de patrimonio técnico, en caso de las obligaciones convertibles en acciones. Por lo tanto estas negociaciones no han ayudado a dinamizar realmente nuestro mercado de valores siendo en muchos casos simplemente un aporte estadístico para los volúmenes negociados.

No se debe desconocer la participación permanente del sector público en las negociaciones en el mercado de valores, a pesar que a partir del 31 de diciembre de 2008, se reformó el Art. 37 de la Ley de Mercado de Valores a través de la Ley de Creación de la Red de 


\section{Las empresas públicas con economía mixta en el mercado de valores en el}

Ecuador

Vol. 2, núm. 1., (2018)

Felix Cristobal Hablich Sánchez; Ingrid Irene Toala Rocuano, Mercy Victoria Agila Maldonado

Seguridad Financiera y por lo tanto no siempre existe la obligatoriedad que el sector público negocie en bolsa.

En consecuencia, sobre todo en este año, se percibe una disminución importante de la participación del Estado en las negociaciones bursátiles. Se debe destacar, dentro de los inversionistas compradores del sector público y de nuestro mercado de valores en general, al Instituto Ecuatoriano de Seguridad Social (IESS), hoy Banco del IESS (BIESS), a lo largo de la historia de nuestro país. Este inversionista institucional, que ante todo compra valores en un mercado primario y espera los vencimientos de los plazos de sus inversiones, como podemos apreciar en el siguiente gráfico, ha tenido una importantísima participación sobre el total negociado cada año. La excepción está dada en los últimos períodos por su considerable disminución de participación en el mercado bursátil debido a la reforma ya indicada sobre la obligación legal que las instituciones públicas siempre inviertan en valores a través del mercado bursátil. Si es que un mercado de valores presenta pocos emisores y pocos inversores, la consecuencia natural es que su peso en la economía nacional sea muy bajo. Por lo que esta escasa participación del volumen de las negociaciones bursátiles se refleja en un pobre \% del PIB de nuestro país y también a nivel internacional (Lovato Saltos, 2013, pág. 225, 226 y 227).

No existen límites mínimos para la participación en el mercado bursátil, pero es más importante reconocer que emitir o abrir su capital en Bolsa implica trabajar de una forma diferente, con mayor orden, transparencia de cuentas, balances auditados, etc. Sobretodo el reconocimiento del gobierno corporativo como una forma de operar porque la propiedad de la empresa se comparte en el caso de las acciones - renta variable- o se debe mostrar solidez para 


\section{Las empresas públicas con economía mixta en el mercado de valores en el Ecuador}

Vol. 2, núm. 1., (2018)

Felix Cristobal Hablich Sánchez; Ingrid Irene Toala Rocuano, Mercy Victoria Agila Maldonado

poder hacer frente a las obligaciones, en el caso de las obligaciones-renta fija. Los requisitos para emitir:

- Solicitar a la Superintendencia de Compañías su inscripción al Registro de Mercado de Valores.

- Publicar en un diario de la localidad, la resolución de inscripción de la compañía en el Registro de Mercado de Valores.

- Elaboración de un prospecto de oferta pública primaria, en donde se indicará la actividad de la empresa, estados financieros, características de la emisión, calificación de riesgo y otros elementos importantes para el futuro inversionista.

- Inscribirse en la Bolsa de valores como emisor, previa resolución de su directorio.

- Identificar la casa de valores que lo acompañará como bróker en su colocación para asegurar su gestión efectiva.

- Cancelar cuota de inscripción para negociar sus títulos en la Bolsa de Valores. (Prado \& Mariela, 2016, pág. 3).

\section{Conclusiones.}

El Estado, dentro de este proyecto de democratización sustantiva, deja de absorber a lo público para transformarse en un núcleo regulador, en el que las distintas alternativas generadas en la sociedad pueden tener expresión. El Estado, así, se descongestiona de demandas y de poderes, que pasan a ser autoadministrados por la comunidad, sin transformarse en parte del mundo de la mercancía. La forma de esta democratización en lo económico y social es la 


\section{Las empresas públicas con economía mixta en el mercado de valores en el}

Ecuador

Vol. 2, núm. 1., (2018)

Felix Cristobal Hablich Sánchez; Ingrid Irene Toala Rocuano, Mercy Victoria Agila Maldonado

cogestión, la autogestión, la cooperativización, que crean entre lo privado y lo estatal un espacio de socialización, de descentralización y de autonomización de las decisiones.

Esta economía de estructura mixta, bajo control de la sociedad, exige un tipo de organización política que acerque a representantes y representados, que desburocratice la gestión y la haga más transparente, que incremente la participación del ciudadano. Todo ello implica reformas institucionales profundas, que abarcan desde la organización del Estado hasta la modernización del sistema de partidos y el fortalecimiento del poder de éstos frente a las corporaciones y los «anillos burocráticos» que penetran en la administración gubernamental. En muchos países esos cambios suponen una reforma de las Constituciones vigentes (Portantiero, 2013, pág. 167).

El mercado de valores ha tenido ciertas variaciones en los montos negociados anualmente, según un breve análisis Bursátil de la evolución de este Mercado realizado durante el año 2012 manifiesta que la capitalización bursátil se mantiene en un constante crecimiento. El Mercado de Valores Ecuatoriano se desempeña como un organismo base que tiene como propósito corroborar el correcto funcionamiento de grandes entidades financieras del Estado, regulando entidades elementales como lo es la Bolsa de Valores de Guayaquil, Bolsa de Valores de Quito, Casas de Valores, El Decevale entre otros, tiene un bajo crecimiento debido a la falta de implementación de mecanismos de captación de nuevas empresas y de sus formas de operar El desconocimiento sobre las transacciones que se operan en un mercado de valores es debido al desinterés de invertir en el mismo. Existe una limitada capacidad de decisión para crear acciones y ampliar el capital. A su vez la falta de conocimiento sobre análisis técnico para saber cómo 


\section{Las empresas públicas con economía mixta en el mercado de valores en el Ecuador}

Vol. 2, núm. 1., (2018)

Felix Cristobal Hablich Sánchez; Ingrid Irene Toala Rocuano, Mercy Victoria Agila Maldonado invertir en un mercado de valores, causa un bajo nivel de rentabilidad por no manejar adecuadamente las inversiones, por lo que las empresas se rehúsan a contraer un riesgo, a corto o largo plazo, en la compra de productos en un mercado de valores evitando pérdidas por una mala administración de los ahorros o excedentes de dinero que decidan invertir. (Pérez, Rivera, \& Solís, 2015, pág. 9, 10, 11, 14 y 15).

El mercado en sí mismo se encuentra limitado por sus propios agentes, la libertad de mercado no ha sido eficiente para dinamizar una actividad que procura el bienestar común reduciendo costos de financiamiento, incentivando la inversión productiva y el ahorro a largo plazo, beneficios relevantes del fortalecimiento del mercado de valores. Por ello, se requiere la intervención efectiva de políticas del regulador, Consejo Nacional de Valores coordinadas con SRI, cartera de economía y producción (Prado \& Mariela, 2016)

Aunque el Estado ha tenido signos de impulsar el Mercado de Valores, la conclusión respecto a la participación de las empresas públicas en el mismo se centra en la confianza. Es preciso la procedencia o el fin del capital el que retiene la decisión del destino de las rentas. De haber una fortaleza real del mundo bursátil ecuatoriano, las empresas públicas mismas deberían ser las primeras obligadas a destinar parte de sus rentas al impulso y fortalecimiento del sistema bursátil.

Por lo tanto, se pueden desarrollar estrategias para la participación en el Mercado de Valores, pero atendiendo el primer paso para la planificación, la investigación, la decisión para el razonamiento economicista sería que aún no existe una variable de confianza que sostenga la 


\section{Las empresas públicas con economía mixta en el mercado de valores en el}

Ecuador

Vol. 2, núm. 1., (2018)

Felix Cristobal Hablich Sánchez; Ingrid Irene Toala Rocuano, Mercy Victoria Agila Maldonado

responsabilidad que implica la inversión del capital mixto de una empresa pública en un mercado de valores con las debilidades que muestra.

Entendiendo que el Estado maneja la economía que sustenta a todo un país y regula las transacciones económicas, no puede dar un uso irresponsable de sus bienes puesto que trae como consecuencia el quiebre de la economía y con él consecuencias sociales de envergadura poniendo en riesgo la gobernabilidad y sostenibilidad del Estado mismo.

\section{Bibliografía.}

Bustamante, M., \& Bustamante, C. (2017). Modelo de desarrollo organizacional de las empresas públicas para impulsar los proyectos de desarrollo socio económico en la provincia de Manabí (Ecuador(. Espacios, 9.

Lovato Saltos, J. I. (2013). Propuesta de reformas al mercado de valores ecuatoriano. IurIs dIctIo , 221-245.

Pérez, O., Rivera, A., \& Solís, L. (2015). Análisis del Mercado de Valores Ecuatoriano como fuente de inversión para las PyMES. Revista Ciencia UNEMI , 8-15.

Portantiero, J. (2013). La múltiple transformación del Estado latinoamericano. Nueva Sociedad (104), 88-94.

Prado, M., \& Mariela, S. (2016). Mercado de Valores ecuatoriano, sus limitantes de desarrollo en el 2015. FCSHOPINA, 88.

Rosero, L. (2010). El desarrollo del mercado de valores en el Ecuador: una aproximación. Ecuador Debate, 23-34. 